\title{
THE CONCIRCULAR VECTOR FIELDS
}

\author{
POOJA S. SAXENA
}

DIT University, Dehradun, India

\section{ABSTRACT}

The aim of the present paper is to investigate the Riemannian manifolds which admit the concircular vector fields. Also investigate some special Finsler Spaces under the assumption that they admit the concircular vector fields. KEYWORDS: Finsler \& Concircular Vector

Received: Dec 13, 2018; Accepted: Jan 03, 2019; Published: Jan 18, 2019; Paper Id.: IJMCARJUN20192

\section{INTRODUCTION}

The notion of the concircular vector fields on a Riemannian manifold $\mathrm{M}$ as vector fields has been introduced by A. Fialkow [13], which satisfy

(1.1) $\quad \nabla_{\mathrm{X}} \mathrm{v}=\mu \mathrm{X}, \mathrm{X} \in \mathrm{TM}$

Where TM is the tangent bundle of $\mathrm{M}, \nabla$ is the Levi-Civita connection and $\mu$ is a non-trivial function on M. If in (1.1) the function $\mu$ is one then a concircular vector field ' $\mathrm{v}$ ' is called a concurrent vector field.

Different types of recurrence are discussed, some important special Finsler spaces is investigated: C-reducible, Semi-C- reducible, quasi - C-reducible, C2-like, S3-like, R-reducible, $\mathrm{T}^{\mathrm{h}}$-recurrent etc.

\section{RIEMANNIAN MANIFOLDS WITH THE CONCIRCULAR VECTOR FIELDS}

Theorem 2.1.:[1] If M is a Riemannian n-manifold which admits a nowhere zero concircular vector field, then $M$ is locally a warped product $I \times_{\varphi(s)} F$, where $\varphi(s)$ is a nowhere vanishing function and $F$ is a Riemannian (n-1)-manifold.

Proof: Assume that on a Riemannian manifold there is a concircular vector field $\mathrm{v}$ such that it is nowhere zero. Let us put

$$
\text { (2.1) } \mathrm{v}=\varphi \mathrm{e}_{1}
$$

Where $e_{1}$ is a unit vector field in the direction of $v$. Extend $e_{1}$ to an orthonormal frame $e_{1}, \ldots \ldots \ldots e_{n}$ on M.It follows from (1.1) and a direct computation that

(2.2) $\mathrm{R}\left(\mathrm{e}_{\mathrm{i}}, \mathrm{v}\right) \mathrm{v}=\nabla_{\mathrm{e}_{\mathrm{i}}} \nabla_{\mathrm{v}} \mathrm{v}-\nabla_{\mathrm{v}} \nabla_{\mathrm{e}_{\mathrm{i}}} \mathrm{v}-\nabla_{\left[\mathrm{e}_{\mathrm{i}, \mathrm{v}]}\right.} \mathrm{v}$

$=\left(e_{i} \mu\right) v-(v \mu) e_{i}, \quad i=2, \ldots \ldots, n$,

Where $\mu$ is defined by (1.1). From (2.2) and $\left\langle R\left(\mathrm{e}_{\mathrm{i}}, \mathrm{v}\right) \mathrm{v}, \mathrm{v}\right\rangle=0$, we get

(2.3) $\mathrm{e}_{2} \mu=\cdots . .=\mathrm{e}_{\mathrm{n}} \mu=0$

Thus the gradient $\nabla \mu$ is parallel to $\mathrm{v}$. 
From (1.1) with $\mathrm{X}=\mathrm{e}_{1}$ and (2.1) we find

$\mu \mathrm{e}_{1}=\nabla_{\mathrm{e} 1}\left(\varphi \mathrm{e}_{1}\right)=\left(\mathrm{e}_{1} \varphi\right) \mathrm{e}_{1}+\varphi \nabla_{\mathrm{e} 1} \mathrm{e}_{1}$

Which gives

(2.4) $\mathrm{e}_{1} \varphi=\mu, \nabla_{\mathrm{e} 1} \mathrm{e}_{1}=0$

From the second equation in (2.4) we know that the integral curves of $e_{1}$ are geodesics in M. Therefore the distribution $D_{1}=\operatorname{Span}\left\{\mathrm{e}_{1}\right\}$ is a totally geodesic foliation. Let us put

$D_{2}=\operatorname{Span}\left\{\mathrm{e}_{2}, \ldots . \mathrm{e}_{\mathrm{n}}\right\}$

From (2.1) and (1.1) with $\mathrm{X}=\mathrm{e}_{\mathrm{i}}$ with $\mathrm{i}=2, \ldots, \mathrm{n}$, we find

$\mu \mathrm{e}_{\mathrm{i}}=\nabla_{\mathrm{e}_{\mathrm{i}}}\left(\varphi \mathrm{e}_{1}\right)=\left(\mathrm{e}_{\mathrm{i}} \varphi\right) \mathrm{e}_{1}+\varphi \nabla_{\mathrm{e}_{\mathrm{i}}} \mathrm{e}_{1}$,

Which implies

$$
\begin{aligned}
& \mathrm{e}_{2} \varphi=\cdots .=\mathrm{e}_{\mathrm{n}} \varphi=0, \\
& \varphi \nabla_{\mathrm{e}_{\mathrm{i}}} \mathrm{e}_{1}=\mu \mathrm{e}_{\mathrm{i}}
\end{aligned}
$$

It follows from

$\nabla_{X} e_{i}=\sum_{j=1}^{n} \omega_{i}^{j}(X) e_{j}, \quad i=1, \ldots . n$

And (2.6) that

(2.7) $\omega_{\mathrm{i}}^{1}\left(\mathrm{e}_{\mathrm{j}}\right)=\frac{\mu}{\varphi} \delta_{\mathrm{ij}}, 2 \leq \mathrm{i}, \mathrm{j} \leq \mathrm{n}$

From (2.7) we conclude that $\mathrm{D}_{2}$ is an integrable distribution whose leaves are totally umbilical in M. Moreover, the mean curvature of leaves of $D_{2}$ are given by $\mu / \varphi$. Since leaves of $D_{2}$ are hypersurfaces, it follows from (2.3) and (2.5) that the mean curvature vector fields of $D_{2}$ are parallel in the normal bundle in $M$. Thus $D_{2}$ is a spherical foliation. Consequently, as we consider the vector field is given byv $=\varphi(\mathrm{s}) \frac{\partial}{\partial \mathrm{s}}$, [6]we conclude that $\mathrm{M}$ is locally a warped product $\mathrm{I} \times_{\mathrm{f}(\mathrm{s})} \mathrm{F}$, where $\mathrm{f}(\mathrm{s})$ is a function on $\mathrm{I}, \partial / \partial \mathrm{s}=\mathrm{e}_{1}$, and $\mathrm{F}$ is a Riemannian (n-1)-manifold. Therefore the sectional curvature of $M$ satisfies

(2.8) $K\left(e_{1}, X\right)=-\frac{f^{\prime \prime}(s)}{f(s)}$

for any unit vector $\mathrm{X}$ orthogonal to $\mathrm{e}_{1}$.

On the other hand, it follows from (2.1) and (2.2) that we also have

(2.9) $\varphi \mathrm{K}\left(\mathrm{e}_{1}, \mathrm{X}\right)=-\mathrm{v} \mu=-\mu^{\prime}(\mathrm{s})$

For any unit vector $X$ orthogonal to to $e_{1}$. Thus, after combining (2.9) with (2.4) and (2.8), we obtain

$$
\frac{\mathrm{f}^{\prime \prime}(\mathrm{s})}{\mathrm{f}(\mathrm{s})}=\frac{\mu^{\prime}(\mathrm{s})}{\varphi}=\frac{\varphi^{\prime \prime}(\mathrm{s})}{\varphi(\mathrm{s})}
$$

Thus if we choose $\mathrm{f}(\mathrm{s})=\varphi(\mathrm{s})$, then $\mathrm{M}$ is locally a warped product $\mathrm{I} \times_{\varphi(\mathrm{s})} \mathrm{F}$. A direct computation yields 
$\nabla_{\mathrm{X}}\left(\varphi(\mathrm{s}) \frac{\partial}{\partial \mathrm{s}}\right)=\varphi^{\prime}(\mathrm{s}) \mathrm{X}$ for $\mathrm{X}$ tangent to I $\times_{\mathrm{f}(\mathrm{s})} \mathrm{F}$.

\section{SPECIAL FINSLER SPACES ADMITTING CONCIRCULAR $\pi$-VECTOR FIELDS}

Special Finsler manifolds arise by imposing extra conditions on the torsion tensors and curvature available in the space. In the context of Finsler geometry, due to the abundance of such geometric objects, there are numerous special Finsler Spaces. From global point of view, a complete and systematic study of special Finsler space has been accomplished in[4]. We investigate the effect of the existence of a concircular $\pi$-Vector field on some important special Finsler spaces. The intrinsic definitions of the special Finsler spaces treated here are quoted from [4].

Theorem 3.1.[2] If a Finsler space admits a concircular vector field then each of the following spaces are Riemannian

- Landsberg space

- 2- dimensional Finsler space

- 3-dimensional Finsler space satisfying condition of Brickell's theorem

- $\quad$ - reducible Finsler space

- Finsler space of $(\alpha, \beta)$ metric

Theorem 3.2. [5] Let $(M, L)$ be a Finsler manifold and $g$ the Finsler metric defined by $L$. There exist a unique regular connection $\nabla$ on $\pi^{-1}(T M)$ such that

- $\quad \nabla$ is metric $: \nabla g=0$;

- $\quad$ The (h) h-torsion of $\nabla$ vanishes : $Q=0$;

- The (h) $h v$-torsion $T$ of $\nabla$ satisfies:

$\mathrm{g}(\mathrm{T}(\overline{\mathrm{X}}, \overline{\mathrm{Y}}), \overline{\mathrm{Z}})=\mathrm{g}(\mathrm{T}(\overline{\mathrm{X}}, \overline{\mathrm{Z}}), \overline{\mathrm{Y}})$

Such a connection is called the Cartan connection associated with the Finsler manifold (M, L)

Theorem 3.3. [3]Let $(M, L)$ be a Finsler manifold admitting a concircular $\pi-v e c t o r$ field $\bar{\zeta}$. Then we have:

The concircular $\pi$-vector field $\bar{\zeta}$ is everywhere non zero.

- The scalar function $B:=g(\bar{\zeta}, \bar{\eta})$ is everywhere non-zero

- $\quad$ The $\pi$-vector field $\overline{m:}=\bar{\zeta}-\frac{B}{L^{2}} \bar{\eta}$ is everywhere non-zero and is orthogonal to $\bar{\eta}$

- $\quad$ The $\pi$-vector field $\bar{m}$ and $\bar{\zeta}$ satisfy $g(\bar{m}, \zeta)=g(\bar{m}, \bar{m}) \neq 0$.

- The scalar function $h(\bar{\zeta}, \bar{\zeta})$ is everywhere non-zero.

Proof:

(a) Follows by Theorem 3.2

(b) Suppose that $B:=g(\bar{\zeta}, \bar{\eta})=0$, then 
$0=\left(\nabla_{\gamma \bar{x}} \mathrm{~g}\right)(\bar{\zeta}, \bar{\eta})=\nabla_{\gamma \bar{x}} \mathrm{~g}((\bar{\zeta}, \bar{\eta})-\mathrm{g}(\bar{\zeta}, \overline{\mathrm{X}})$

$=-\mathrm{g}((\bar{\zeta}, \overline{\mathrm{X}}), \forall \overline{\mathrm{X}} \in(\pi(\mathrm{M}))$

Hence, as $\mathrm{g}$ is non-degenerate, $\zeta$ vanishes, which contradicts (a). Consequently, $\mathrm{B} \neq 0$

(c) If $\bar{m}=0$, then $L^{2} \bar{\zeta}-B \bar{\eta}=0$. Differentiating covariantly with respect to $\gamma \bar{X}$, we get

(3.1) $2 \mathrm{~g}(\overline{\mathrm{X}}, \bar{\eta}) \bar{\zeta}-\mathrm{B} \overline{\mathrm{X}}-\mathrm{g}(\overline{\mathrm{X}}, \bar{\zeta}) \bar{\eta}=0$

From which,

$$
\mathrm{g}(\overline{\mathrm{X}}, \bar{\zeta})=\frac{\mathrm{B}}{\mathrm{L}^{2}} \mathrm{~g}(\overline{\mathrm{X}}, \bar{\eta})
$$

(3.1), using (3.2), we obtain

$$
\begin{aligned}
& 0=2 g(\bar{X}, \bar{\eta}) g(\bar{Y}, \bar{\zeta})-\operatorname{Bg}(\bar{X}, \bar{Y})-\quad g(\bar{X}, \bar{\zeta}) g(\bar{Y}, \bar{\eta}) \\
& =2 \frac{B}{L^{2}} g(\bar{Y}, \bar{\eta}) g(\bar{X}, \bar{\eta})-\operatorname{Bg}(\bar{X}, \bar{Y})-\quad \frac{B}{L^{2}} g(\bar{X}, \bar{\eta}) g(\bar{Y}, \bar{\eta}) \\
& =-B\left\{g(\bar{X}, \bar{Y})-\frac{1}{L^{2}} g(\bar{Y}, \bar{\eta}) g(\bar{X}, \bar{\eta})\right\} \\
& =-B h(\bar{X}, \bar{Y})
\end{aligned}
$$

Since $B \neq 0$, we are led to a contradiction: $h=0$. Consequently, $\overline{\mathrm{m}} \neq 0$.

On the other hand, the orthogonality of the two $\pi$ - vector fields $\bar{m}$ and $\bar{\eta}$ follows from the identities $g(\bar{\eta}, \bar{\eta})=$ $\mathrm{L}^{2}$ and $\mathrm{g}(\bar{\eta}, \bar{\zeta})=\mathrm{B}$
(a) Follows from (c)
(b) Follows from (d), (c) and the fact that $h(X, \eta)=h(\eta, X)=0$.

\section{REFERENCES}

1. Bang-Yen Chen, Bull. Korean Math.Soc.52(2015), No.5, pp 1535-1547

2. B.N. Prasad, V.P. Singh and Y.P. Singh, Indian J. pure appl. Math.,17(8): 998-1007, August 1986

3. Nabil L. Youssef, Amr Soleiman, Advances in Pure Mathematics, 2013,3,282-291

4. N.L. Youssef, S.H. Abed and A. Soleiman, "A Global approach to the Theory of Special Finsler Manifolds", Kyoto Journal of Mathematics, Vol.48, no.4,2008, pp.857-893

5. N.L. Youssef, S.H. Abed and A. Soleiman," Cartan and Berwald connections in the Pullback Formalism," Algebras, Groups and Geometries, Vol.25, No.4,2008pp 363-386

6. T. Poorna Kantha, A.S.N. Murti \& V.V. Ramana Rao, Secondary Flow of two Immiscible Liquids in a Rotating Annular Pipe of Circular Cross Section (IJAMSS), Volume 1, Issue 2, November 2012, pp. 37-49

7. S.Hieko, Eineinnere Kennzeichnungderverzerrten Produkte, Math. Ann.241(1979)no. 3, 209-215 